\title{
Modo de ação de fosfitos de potássio no controle da podridão olho de boi em maçã
}

\author{
Piérri Spolti ${ }^{1}$, Rosa Maria Valdebenito-Sanhueza ${ }^{2}$, Angela Diniz Campos $^{3}$, Emerson Medeiros Del Ponte $^{1}$
} ${ }^{1}$ Departamento de Fitossanidade, Faculdade de Agronomia, Universidade Federal do Rio Grande do Sul, Av. Bento Gonçalves, 7712 , CEP 91540000,
Porto Alegre, RS. ${ }^{2}$ Proterra Consultoria Agronômica, BR 116, 7320, Bairro Fátima, CEP 95200-000, Vacaria, RS. Pesquisadora CNPq. ${ }^{3}$ Embrapa
Clima Temperado, Caixa Postal 403, CEP 96001-970, Pelotas, RS.

Autor para correspondência: Rosa Maria Valdebenito-Sanhueza (rosamaria@cipnet.com.br)

Data de chegada: 06/03/2014. Aceito para publicação em: 16/01/2015.

$10.1590 / 0100-5405 / 1982$

\section{RESUMO}

Spolti, P.; Valdebenito-Sanhueza, R. M.; Campos, A. D.; Del Ponte, E. M. Modo de ação de fosfitos de potássio no controle da podridão olho de boi em maçã. Summa Phytopathologica, v.41, n.1, p.42-48, 2015.

A podridão olho de boi causada por Cryptosporiopsis perennans é a principal doença de maçãs em pós-colheita no Brasil. Objetivou-se neste trabalho avaliar a) o uso de sais de fosfito de potássio em associação ou não ao fungicida captan no controle da doença em condições de campo e in vitro, b) as modificações físico-químicas nos frutos e, c) o efeito desses sais na atividade da peroxidase e da fenilalanina amonia-liase em maçãs inoculadas artificialmente. As aplicações dos sais de fosfito de potássio e captan foram realizadas na safra 2007/08, município de Vacaria, em pomar da cv. Pink Lady $^{\circledR}$ a partir dos 45 dias antes da colheita ou $24 \mathrm{~h}$ antes da colheita. A atividade da peroxidase e da fenilalanina amônia-liase foi determinada em frutos 24 h e 14 dias após as inoculações com o patógeno. Aplicações de fosfito de potássio + captan reduziram em $60 \%$ os danos pela podridão olho de boi em pós-colheita, além de reduzir $66 \%$ da quantidade de inóculo na superfície das maçãs em aplicação realizada no dia anterior à colheita. O fosfito de potássio inibiu o desenvolvimento in vitro do fungo sem alterar a atividade das enzimas peroxidase e fenilalanina amônia-liase. Os fosfitos de potássio mostraram ação fungicida erradicante, com potencial de uso no manejo integrado da podridão olho de boi.

Palavras-chave adicionais: pós-colheita, fenilalanina amônia-liase, peroxidase, Cryptosporiopsis sp., Malus domestica.

\section{ABSTRACT}

Spolti, P.; Valdebenito-Sanhueza, R. M.; Campos, A. D.; Del Ponte, E. M. Mode of action of potassium phosphite on bull's eye rot of apple. Summa Phytopathologica, v.41, n.1, p.42-48, 2015.

The bull's eye rot, caused by Cryptosporiopsis perennans, is the most important postharvest disease of apples in Brazil. The aims of this study were to evaluate a) the use of potassium phosphite salts isolated or in mixture with the fungicide captan for disease control in the field and in vitro; b) the physical/ chemical alterations of fruits and, c) the effect of these salts on peroxidase and phenylalanine ammonia-lyase activity in artificially inoculated apples. Potassium phosphites and captan were applied in the 2007/08 season, at Vacaria City, to an orchard of cv. Pink Lady ${ }^{\circledR}$, starting at 45 days or $24 \mathrm{~h}$ prior to harvest. The activity of peroxidase and phenylalanine ammonia-lyase was measured for apple fruits at $24 \mathrm{~h}$ and 14 days after inoculations with the pathogen. Applications of potassium phosphite + captan reduced by $60 \%$ the postharvest loss caused by bull's eye rot, besides decreasing $66 \%$ of the inocula produced on apple surface when applied on the day prior to harvest. Potassium phosphite inhibited the in vitro growth of the fungus without affecting peroxidase and phenylalanine ammonia-lyase activity. Potassium phosphites showed eradicating fungicide action and potential to be used in the integrated management of bull's eye rot.

Additional keywords: postharvest, phenylalanine ammonia-lyase, peroxidase, Cryptosporiopsis perennans, Malus domestica.

O fungo Cryptosporiopsis perennans (Cp.) (Zeller \& Childs) Wollenweb. (= Gloeosporium perennans Zeller \& Childs), anamorfo de Pezicula perennans (Zeller \& Childs) (sin. Neofabraea perennans), é o agente causal das doenças podridão olho de boi (POB) e cancro da macieira (Malus domestica) no Brasil (22). A POB, relatada no Brasil em 1996, está presente em todas as regiões produtoras de maçã do Rio Grande do Sul, Santa Catarina e Paraná, onde ocasionou danos de até $16 \%$ na cultivar Fuji na fase de armazenamento (22).

Apesar da recomendação de uso de medidas em um sistema integrado de manejo, o controle das podridões em maçãs é feito basicamente com aplicações de fungicidas na fase final de maturação dos frutos (3). O uso excessivo de agrotóxicos nas lavouras é uma crescente preocupação mundial com as possibilidades reais de contaminação ambiental. Efeitos crônicos de contaminação ambiental por pesticidas, em várias regiões do globo, estão interferindo em fatores como expectativa de vida, crescimento, fisiologia, comportamento e reprodução dos seres vivos (24). Uma alternativa é o uso de alguns fertilizantes que são eficientes no controle de fitopatógenos e menos tóxicos que os fungicidas tradicionalmente utilizados (12). Nesse grupo de produtos se encontram os sais de fosfito, que são mais uma opção no manejo integrado de algumas doenças da macieira, como doenças radiculares, foliares ou que causam danos cosméticos nos frutos $(1,20$, 21) e cuja já rota metabólica tem sido estudada em algumas culturas (9).

Os fosfitos de potássio vêm sendo frequentemente utilizado 
em macieira no controle da mancha foliar da gala (Colletotrichum gloeosporioides) e sarna (Venturia inaequalis). No entanto, informações na literatura para o controle de podridões de frutos usando fosfitos de potássio limitam-se a estudos na fase de pós-colheita $(4,5)$. No caso da $\mathrm{POB}$, não há relatos do uso de fosfitos de potássio no controle da doença no campo. Os efeitos fungicida e fungistático dos sais de potássio foram demonstrados para o controle da mancha foliar da gala em macieiras $(1,2)$. Porém, não foi avaliado o potencial efeito dos sais de potássio na produção de enzimas relacionadas à defesa do hospedeiro, que pode ser caracterizado por mudanças na atividade da peroxidase (PO) e fenilalanina amônia-liase (FAL) (8).

Assim, objetivou-se com o presente estudo avaliar o modo de ação e o potencial de uso de sais de fosfito de potássio isoladamente ou em associação com fungicida captan, no controle da podridão olho de boi em condições de campo e in vitro, e o seu efeito na atividade da peroxidase e da fenilalanina amônia-liase em maçãs inoculadas artificialmente.

\section{MATERIAL E MÉTODOS}

\section{Área de estudo e condução dos experimentos}

Os experimentos de campo foram conduzidos em pomar comercial de 12 anos de idade da cultivar Pink Lady ${ }^{\circledR}$ sobre porta-enxerto MM-9 localizado no município de Vacaria-RS, numa densidade de 2.857 plantas por hectare. Os experimentos em condições controladas foram realizados no Laboratório de Fitopatologia da Estação Experimental da Embrapa Uva e Vinho, do mesmo município. As análises enzimáticas foram executadas no Laboratório de Fisiologia Vegetal na Embrapa Clima Temperado, Pelotas-RS.

\section{Avaliação de efeito dos sais de fosfito no controle da POB no} campo

Os tratamentos com o uso de sais de fosfito isoladamente, e/ou em mistura com o fungicida captan foram avaliados em dois períodos: 1) aos 45 dias antes da colheita (com aplicações a cada sete dias, ou quando o acúmulo de precipitação pluvial no período compreendido entre aplicações excedeu $30 \mathrm{~mm}$ ) e, 2) um dia antes da colheita. No primeiro período foram avaliados os tratamentos: a) $2 \mathrm{~mL}$ de fosfito de potássio (40\% $\mathrm{P}_{2} \mathrm{O}_{5}$ e $\left.20 \% \mathrm{~K}_{2} \mathrm{O}\right) . \mathrm{L}^{-1}$ de água ( $400 \mathrm{~g}$ de $\mathrm{P}_{2} \mathrm{O}_{5}$ e $200 \mathrm{~g}$ de $\left.\mathrm{K}_{2} \mathrm{O} \cdot \mathrm{kg}^{-1}\right)$; b) $2 \mathrm{~mL}$ de fosfito de potássio $\left(30 \% \mathrm{P}_{2} \mathrm{O}_{5}\right.$ e $\left.20 \% \mathrm{~K}_{2} \mathrm{O}\right) . \mathrm{L}^{-1} \mathrm{de}$ água; c) $2 \mathrm{~mL}$ de fosfito de potássio $\left(40 \% \mathrm{P}_{2} \mathrm{O}_{5}\right.$ e $\left.20 \% \mathrm{~K}_{2} \mathrm{O}\right) \mathrm{em}$ mistura com $1,2 \mathrm{~g}$ de captan. $\mathrm{L}^{-1}$ de água; d) $2 \mathrm{~mL}$ de fosfito de potássio $(30 \%$ $\mathrm{P}_{2} \mathrm{O}_{5}$ e $20 \% \mathrm{~K}_{2} \mathrm{O}$ ) em mistura com $1,2 \mathrm{~g}$ de captan. $\mathrm{L}^{-1}$ de água 5) 1,2 $\mathrm{g}^{2}$ de captan. $\mathrm{L}^{-1}$ de água e; e) testemunha (controle), sem aplicação de fungicidas desde a instalação do ensaio. A colheita foi realizada na última semana de abril. Nesse experimento com tratamentos protetores iniciados aos 45 dias anteriores à colheita foram feitas cinco aplicações de sais de fosfito de potássio ou com o fungicida protetor.

No segundo período foi avaliado o efeito de uma única pulverização, $24 \mathrm{~h}$ antes à colheita, de $2 \mathrm{~mL}$ de fosfito de potássio $\left(40 \% \mathrm{P}_{2} \mathrm{O}_{5}\right.$ e $20 \%$ $\mathrm{K}_{2} \mathrm{O}$ ) misturado com 1,2 g de captan. $\mathrm{L}^{-1}$ de água, em comparação com a testemunha (sem pulverização). A colheita foi realizada na primeira semana de junho/2008.

As aplicações foram feitas com o auxílio de um pulverizador costal manual com vazão de 0,55 L.planta ${ }^{-1}$, atingindo o ponto de escorrimento, tendo-se um volume de parte aérea de $3 \mathrm{~m}^{3}$. Não foram utilizados surfactantes.

$\mathrm{Na}$ colheita, 90 frutos amostrados aleatoriamente nas parcelas experimentais foram avaliados quanto à incidência de POB $(\%$ de frutos com sintomas de podridão). Dos frutos assintomáticos, 30 foram avaliados visando à detecção de infecção latente, imergindo os frutos em água por $6 \mathrm{~h}$ e a seguir incubando-os a $25^{\circ} \mathrm{C}$. A incidência de infecções latentes (\%) de POB foi avaliada 30 dias após o início da incubação (DAI). Os demais frutos assintomáticos foram armazenados em câmara frigorífica $\left(3^{\circ} \mathrm{C}\right.$ e $95 \%$ UR do ar) durante três meses para uma última avaliação da incidência de POB. O experimento de campo foi conduzido em blocos casualizados com quatro repetições. Cada parcela experimental foi composta por seis plantas, com parcela útil de quatro plantas centrais.

Efeito de fosfitos sobre o inóculo de Cryptosporiopsis perennans. em maçãs do campo

Frutos assintomáticos (8 frutos por parcela) foram amostrados aleatoriamente por ocasião da colheita. Esses frutos foram lavados com água destilada esterilizada (ADE) e surfactante não-iônico (Tween $0,001 \%$ ) submetidos à sonicação (dispersão das partículas por ultrasom) durante 1 min. Na sequência, uma alíquota da solução resultante da lavagem foi distribuída em placas de Petri com meio semiseletivo para $C$ p. $(19,23)$.

As placas foram então incubadas em câmara de crescimento por 21 dias sob fotoperíodo de $12 \mathrm{~h}$ e temperatura de $22{ }^{\circ} \mathrm{C}$. Após, foi registrado o número de unidades formadoras de colônias (UFC) de $C$ p. nas placas. $\mathrm{O}$ experimento foi conduzido em blocos casualizados com quatro repetições. Cada repetição foi composta por oito placas de Petri (uma placa de Petri/fruto amostrado na parcela).

Efeito de fosfitos no crescimento in vitro de Cryptosporiopsis perennans.

O método usado foi o descrito para Colletotrichum gloeosporioides (2) nas concentrações de $0 ; 0,0625 ; 0,125 ; 0,250$ e $0,500 \mu \mathrm{L}$ de fosfito de potássio $\left(40 \% \mathrm{P}_{2} \mathrm{O}_{4}\right.$ e $\left.20 \% \mathrm{~K}_{2} \mathrm{O}\right) / \mathrm{mL}$ de BDA corrigido para $\mathrm{pH} 2$ ou $\mathrm{pH} 7$ em placas de Petri de $90 \mathrm{~mm}$ de diâmetro com aproximadamente $20 \mathrm{~mL}$ do meio por placa. O diâmetro das colônias (mm) foi medido diariamente para estimar o índice de velocidade de crescimento micelial (IVCM), expresso em mm/dia (2). Adicionalmente, uma alíquota de uma suspensão na concentração de $1 \times 10^{2}$ conídios $/ \mathrm{mL}$ foi distribuída em placas de Petri contendo BDA, com as mesmas concentrações de fosfito de potássio e $\mathrm{pH}$ anteriores, e incubadas por sete dias. Após esse período, foi avaliado o número de UFC, cujos resultados permitiram estimar a germinação dos conídios (\%). O ensaio in vitro foi conduzido em delineamento inteiramente casualizado. Para cada concentração de fosfito de potássio em BDA e combinação de $\mathrm{pH}$ foram utilizadas cinco repetições. $\mathrm{O}$ experimento foi repetido três vezes. Como não houve diferença entre os experimentos, as médias dos três experimentos foram agrupadas para análise estatística.

\section{Análises físico-químicas}

Os frutos armazenados por 30 dias foram analisados quanto ao: a) teor de sólidos solúveis totais (SST - ${ }^{\circ}$ Brix), utilizando-se um refratômetro manual a partir da leitura do suco de 10 frutos por repetição; b) firmeza de polpa após a retirada da casca em dois pontos por fruta (índices de firmeza de polpa nas faces exposta e não exposta ao sol), também em dez maçãs por repetição, utilizando penetrômetro manual com ponteira de $11 \mathrm{~mm}$; c) teor de amido determinado pela reação iodo-amido, em cinco frutos por repetição; d) $\mathrm{pH}$ em medidor de $\mathrm{pH}$ (Digimed DMPH 2) em uma solução de água destilada, contendo $10 \%$ do suco de cinco maçãs para cada repetição; e) acidez titulável por titulação potenciométrica até $\mathrm{pH} 8,1$ na mesma solução utilizada para a determinação do $\mathrm{pH}$, e expressa 
em \% de ácido málico (6). O experimento foi conduzido em blocos casualizados com quatro repetições.

\section{Preparo das amostras para as análises enzimáticas}

Os frutos utilizados foram previamente armazenados por 30 dias em temperatura ambiente. As análises foram realizadas na polpa incluindo a casca da maçã e sempre no lado oposto à inoculação, a qual foi realizada pela deposição de disco de micélio de um isolado de $C$ p. cultivado por sete dias em BDA, sobre uma lenticela e sem ferir o fruto. A amostra consistiu da retirada de um corte vertical da casca até o centro da fruta, em fatias de um grama, sendo esta imediatamente congelada à $-25^{\circ} \mathrm{C}$. As coletas para as avaliações foram realizadas $24 \mathrm{~h}$ e 14 dias após as inoculações. Em ambos os casos, utilizou-se como controle negativo, frutos não inoculados.

\section{Análise da atividade de peroxidase (PO)}

As amostras para análises enzimáticas foram homogeneizadas utilizando banho de gelo em microtriturador à temperatura máxima de $4^{\circ} \mathrm{C}$ em $10 \mathrm{~mL}$ de tampão fosfato $0,05 \mathrm{M}$ (pH 7), contendo $1 \mathrm{mg}$ de polivinilpirrolidona-10. O homogeneizado foi filtrado em papel Whatman $\mathrm{n}^{\circ} 1 \mathrm{e}$ em seguida centrifugado a $4000 \mathrm{~g}$ por $20 \mathrm{~min}$ sob refrigeração e o precipitado descartado. O sobrenadante foi conservado em gelo e usado para as determinações da atividade da PO segundo metodologia descrita por Campos \& Silveira (7). A absorbância foi lida em $450 \mathrm{~nm}$, em espectrofotômetro UV PC Shimadzu. No branco, o extrato enzimático foi substituído por água deionizada. A atividade da PO foi expressa em unidade de enzima por grama de tecido por minuto. Uma unidade da enzima foi definida como a quantidade de extrato enzimático que acusou aumento na absorbância de 0,001 unidade. min $^{-1}$.

\section{Análise da atividade da fenilalanina amônia-liase (FAL)}

A atividade da FAL foi determinada de acordo com os métodos descritos por Hyodo \& Yang, (10) e Hyodo et al. (11), padronizada para análise de frutas conforme modificações de acordo com Campos \& Silveira (7). Brevemente, $1 \mathrm{~g}$ de polpa foi homogeneizado em microtriturador em uma mistura de $4 \mathrm{~mL}$ de tampão borato de sódio 50 $\mathrm{mM}$ pH 8,5 contendo $25 \mathrm{~g} / \mathrm{L}$ de PVP 10 e $4 \mathrm{~mL} / \mathrm{L}$ de mercaptoetanol e em seguida foi realizada a filtração do extrato bruto em papel Whatman $\mathrm{n}^{\mathrm{o}} 1$. O extrato bruto foi centrifugado por $15 \mathrm{~min}$ na velocidade de 25000 $g$ e o sobrenadante (extrato enzímico) foi filtrado em papel Whatman $\mathrm{n}^{\circ} 1$ e utilizado para análise da atividade da FAL. As leituras foram realizadas em espectrofotômetro UV PC Shimadzu em $290 \mathrm{~nm}$. O ensaio de atividade enzimática dos frutos (PO e FAL) teve delineamento em blocos casualizados com três repetições e arranjado em esquema fatorial $6 \times 2 \times 2$ para $\mathrm{PO}$ (tratamentos em pré-colheita x inoculação com Cp. x data de quantificação) e no esquema $6 \times 2$ para FAL, eliminando neste caso o fator data.

\section{Análise estatística}

Um modelo linear misto foi ajustado aos dados dos experimentos de campo, considerando-se os tratamentos como efeito fixo e os blocos como efeito aleatório. As médias dos tratamentos foram comparadas pelo teste de Waller-Duncan $(\mathrm{K}=100)$. O teste de Waller-Duncan, em comparação a outros testes de comparação múltipla de médias, evita o erro do Tipo I e do Tipo II por reduzir a influência dos valores de F. As médias dos dados de análise de enzimas, quando detectado efeito significativo dos tratamentos, foram discriminadas pela diferença mínima significativa (DMS, $P<0,05$ ). Todas as análises estatísticas foram realizadas no programa estatístico SAS (versão 9, SAS Institute, Cary, NC).

\section{RESULTADOS E DISCUSSÃO}

Com as pulverizações nos 45 dias anteriores à colheita, todos os tratamentos reduziram significativamente o número de propágulos de $C$ p. na superfície das maçãs, destacando-se as misturas de fosfito de potássio com captan (Figura 1A).

Houve uma maior incidência de POB na fase de pós-colheita em comparação ao momento da colheita (Figura 1B). No momento da colheita, a incidência de POB no controle (testemunha) foi inferior a $8 \%$ (Figura 1B) enquanto no mesmo tratamento, após 30 dias de armazenamento a incidência de POB foi de 37\% (Figura 1B).

A incidência de frutos com infecções latentes de POB detectadas 30 dias após a incubação foi reduzida em $35 \%$ pela aplicação dos fosfitos no período final de maturação (Figura 1B). No entanto, não houve benefício no controle da doença como no uso da mistura de fosfitos de potássio com o fungicida captan, indiferente à formulação dos fosfitos no controle dessas infecções (Figura 1B). A partir da quarta aplicação dos fosfitos foram observados sintomas de fitotoxidez pelo estiolamento leve nos ápices dos ramos do ano.

Na pré-colheita, as misturas de fosfito de potássio com captan e as formulações de fosfito de potássio $\left(30 \% \mathrm{P}_{2} \mathrm{O}_{5}\right.$ e $\left.20 \% \mathrm{~K}_{2} \mathrm{O}\right)$ e $(40 \%$ $\mathrm{P}_{2} \mathrm{O}_{5}$ e $20 \% \mathrm{~K}_{2} \mathrm{O}$ ) foram mais eficientes no controle da doença quando comparados ao uso sequencial de captan (Figura 1B).

Na pós-colheita, com proteção permanente a partir dos 45 dias antes da colheita, os tratamentos com fosfitos reduziram em até $65 \%$ a incidência da POB (Figura 1B) e o uso de mistura de captan com os fosfitos apresentou maior efeito na redução da incidência apenas para a formulação $30 \% \mathrm{P}_{2} \mathrm{O}_{4}$ e $20 \% \mathrm{~K}_{2} \mathrm{O}$ (Figura 1B).

No experimento in vitro o índice de velocidade de crescimento micelial (IVCM) apresentou relação de ordem quadrática inversa à concentração de fosfito de potássio no meio de cultura $\left(\mathrm{R}^{2}>0,90 *\right)$ indiferente ao $\mathrm{pH}$ de correção (dados não apresentados). $\mathrm{O}$ número de UFC também foi influenciado pela suplementação de fosfito de potássio ao meio de cultura, com ajuste de um modelo quadrático com o mínimo de colônias por placa na concentração de $0,500 \mu \mathrm{L} / \mathrm{L}$ com pH 2 e pH 7 .

A aplicação de fosfito de potássio $\left(40 \% \mathrm{P}_{2} \mathrm{O}_{5}\right.$ e $\left.20 \% \mathrm{~K}_{2} \mathrm{O}\right)$ misturado ou não com captan, bem como o uso isolado do captan, reduziram o $\mathrm{pH}$ do suco das maçãs. Quanto à acidez titulável, a mistura de fosfito de potássio $\left(40 \% \mathrm{P}_{2} \mathrm{O}_{5}\right.$ e $\left.20 \% \mathrm{~K}_{2} \mathrm{O}\right)$ com captan e, aplicação exclusiva deste, aumentou em $17 \%$ o teor de ácido málico no suco dos frutos (Tabela 1). Os valores de teor de açúcar $\left(14,75 \pm 0,59{ }^{\circ}\right.$ Brix $)$, teor de amido $(2,06 \pm 0,15)$ e firmeza da polpa $(18,70 \pm 0,64 \mathrm{lb})$ não diferiram entre os tratamentos. Os valores obtidos sobre as propriedades químicas das maçãs com e sem tratamento não deferiram dos índices normais para as maçãs (9).As alterações dessas características dos frutos, no presente estudo, não apresentaram correlação com a incidência da podridão ( $\mathrm{r}$ $\leq 0,03 ; P \geq 0,57$ ) (dados não apresentados).

Os tratamentos com captan realizados em pré-colheita não influenciaram a atividade enzimática de PO e FAL nos frutos. Para PO houve interação tripla entre os tratamentos, data de amostragem e inoculação dos frutos com $C$ p., como função do efeito da data de amostragem sobre os valores da atividade de PO, visto a não significância estatística individual do tratamento (T) e inoculação (I).

Na segunda data de avaliação, houve um decréscimo de $20 \%$ na atividade enzimática de PO (Figura 2). Entre as causas de variação relacionadas no experimento na atividade enzimática de FAL, apenas a inoculação com $C$ p. afetou significativamente os valores dessa enzima (Figura 2), onde o confronto com o patógeno determinou uma maior atividade da enzima nos frutos (Figura 2). 

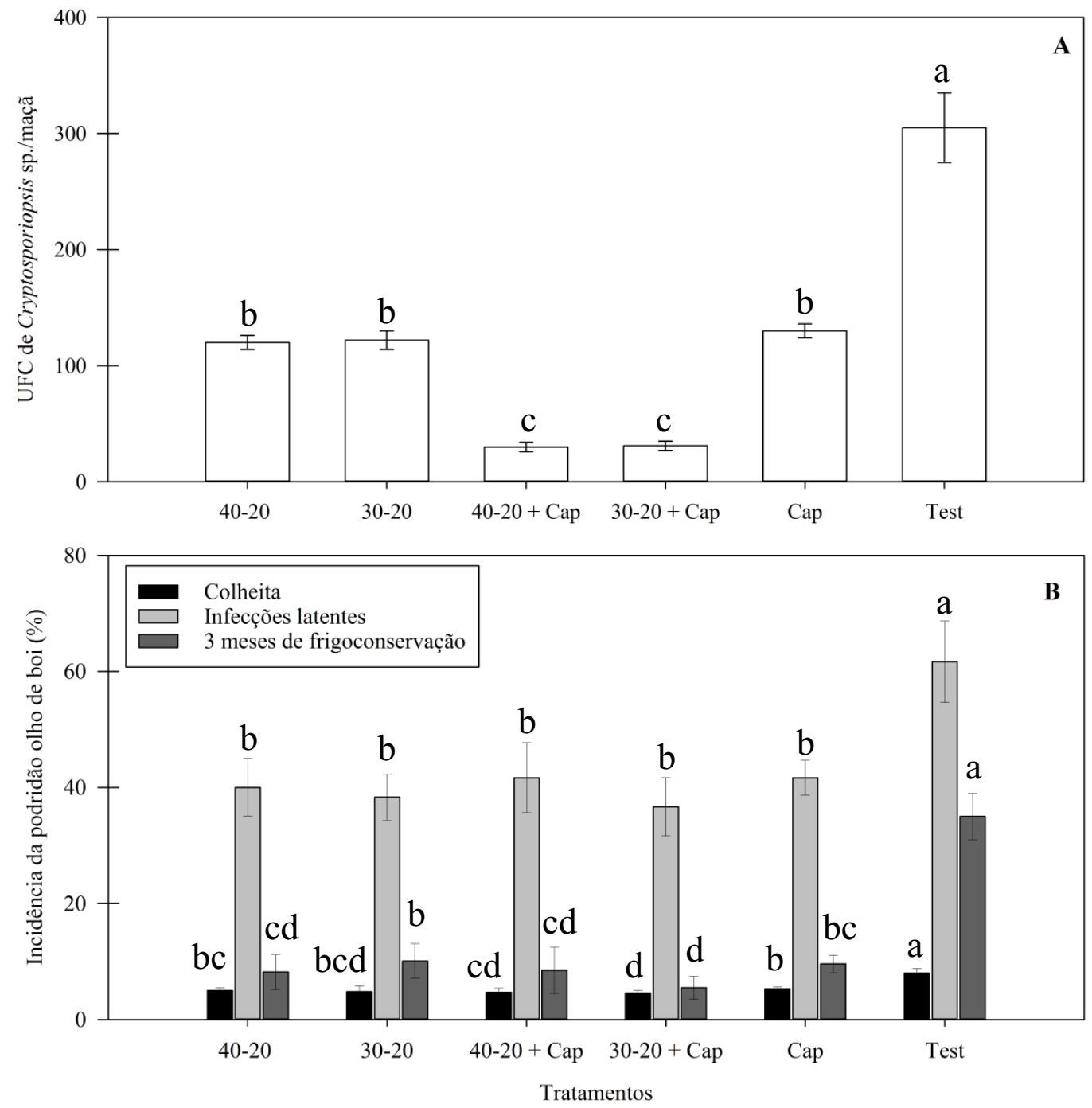

Figura 1. Número de unidades formadoras de colônias (UFC) de Cryptosporiopsis sp. na superfície de maçãs 'Pink Lady ${ }^{\mathbb{E}}$ ' no momento da colheita (A) e incidência da podridão olho de boi no momento da colheita, infecções latentes e após três meses de frigoconservação (B) em maçãs provenientes de parcelas pulverizadas com fosfito de potássio em diferentes combinações de $\mathrm{Pe} \mathrm{K}\left(40 \% \mathrm{P}_{2} \mathrm{O}_{5}+20 \% \mathrm{~K}_{2} \mathrm{O}\right)$ ou $\left(30 \% \mathrm{P}_{2} \mathrm{O}_{5}+20 \% \mathrm{~K}_{2} \mathrm{O}\right)$, aplicados isoladamente ou em mistura com Captan (Cap) e um tratamento controle (Test). Pulverizações realizadas nos 45 dias prévios à colheita. Médias representadas pelas colunas, seguidas por letras distintas, diferem entre $\sin (\mathrm{P}<0,05)$ pelo teste de Waller-Duncan. Para a comparação das médias na Figura 1B comparar dentre cada uma das classes (colheita, infecções latentes ou após o armazenamento). Linhas verticais correspondem ao erro padrão da média. Vacaria, RS. 2007/08.

Tabela 1. Análise química de maçãs cv. Pink Lady ${ }^{\circledR}$ pulverizadas nos 45 dias antecedentes à colheita com os sais de fosfito de potássio e fungicida listados. Vacaria, RS. 2007/08

\begin{tabular}{lcccc}
\hline \multicolumn{1}{c}{ Tratamento } & & pH & \multicolumn{2}{c}{ Acidez titulável } \\
& & & (\% ácido málico) \\
\hline Fosfito de potássio $\left(40 \% \mathrm{P}_{2} \mathrm{O}_{5}+20 \% \mathrm{~K}_{2} \mathrm{O}\right)$ & 3,38 & $\mathrm{BC}^{\mathrm{Y}}$ & 6,98 & $\mathrm{AB}$ \\
Fosfito de potássio $\left(30 \% \mathrm{P}_{2} \mathrm{O}_{5}+20 \% \mathrm{~K}_{2} \mathrm{O}\right)$ & 3,45 & $\mathrm{~A}$ & 6,20 & $\mathrm{~B}$ \\
Fosfito de potássio $\left(40 \% \mathrm{P}_{2} \mathrm{O}_{5}+20 \% \mathrm{~K}_{2} \mathrm{O}\right)+$ captan & 3,34 & $\mathrm{CD}$ & 7,18 & $\mathrm{~A}$ \\
Fosfito de potássio $\left(30 \% \mathrm{P}_{2} \mathrm{O}_{5}+20 \% \mathrm{~K}_{2} \mathrm{O}\right)+$ captan & 3,44 & $\mathrm{AB}$ & 6,60 & $\mathrm{AB}$ \\
Captan & 3,30 & $\mathrm{D}$ & 7,43 & $\mathrm{~A}$ \\
Controle & 3,46 & $\mathrm{~A}$ & 6,13 & $\mathrm{~B}$ \\
\hline C.V. $(\%)$ & & 2,45 & & 5,63 \\
\hline
\end{tabular}

Médias seguidas por letras iguais não diferem entre si pelo teste de Waller-Duncan $(P<0,05)$. 
No experimento com uma única pulverização, $24 \mathrm{~h}$ antes da colheita, a mistura de fosfito de potássio $\left(40 \% \mathrm{P}_{2} \mathrm{O}_{5}\right.$ e $\left.20 \% \mathrm{~K}_{2} \mathrm{O}\right)$ com o captan não reduziu a incidência de POB no momento da colheita (Figura 3). No entanto, a mistura de fosfito de potássio com captan reduziu significativamente os danos ocasionados pela doença em pós-colheita, através da redução da incidência de infecções latentes (Figura 3). Tais efeitos podem ser reflexos da ação erradicante dos compostos avaliados, já que o uso da mistura fosfito de potássio $(40 \%$ $\mathrm{P}_{2} \mathrm{O}_{5}$ e $20 \% \mathrm{~K}_{2} \mathrm{O}$ ) com o captan diminuiu em $66 \%$ do número de UFC sobre os frutos (Figura 3).

A incidência de POB é mais alta na fase de pós-colheita, sendo que, nas condições de campo se restringem aos frutos com ferimentos (22). Tal fato foi ratificado no presente estudo, em que se mostra a importância das infecções latentes na intensidade final da doença. Cultivares de macieira de ciclo longo, a exemplo da cultivar Pink Lady ${ }^{\circledR}$ são particularmente suscetíveis à $\mathrm{POB}$, sendo que a incidência da doença pode exceder $15-30 \%$ após 120 dias de armazenamento, a exemplo do observado no presente estudo.

A redução do número de propágulos na superfície dos frutos está associada ao controle da podridão olho de boi (23). E assim como no presente estudo, a ação erradicante dos sais de fosfito foi também relatada em estudo com zoósporos de Phytophthora cinnamomi (25). Da mesma forma que no controle de oídio (Oidium mangiferae) da mangueira (Mangifera indica) (15), o uso de fosfito de potássio, em mistura ou em alternância com fungicidas de modo de ação diferente, pode aumentar a eficiência de controle de POB, além de reduzir o risco de seleção de isolados resistentes e os custos de produção. Além disto, conforme Rosenberger \& Cox (18), a aplicação o uso de fosfitos de potássio em mistura com captan incrementa a atividade residual desse fertilizante.

Araújo et al. (1) observaram ação curativa de formulações de fosfito de potássio no controle de mancha foliar da gala, com controle de $62 \%$ quando as aplicações foram efetuadas até $48 \mathrm{~h}$ após a inoculação, com destaque para a formulação $40 \% \mathrm{P}_{2} \mathrm{O}_{5}$ e $20 \% \mathrm{~K}_{2} \mathrm{O}$. No presente estudo, o efeito da formulação parece ser secundário quando da utilização ou não de captan em mistura.Segundo Araújo et al. (2) a ação in vitro de sais de fosfitos sobre a fisiologia dos patógenos é um indicativo da ação fungicida direta de tais compostos, assim como observado no presente estudo.

A ausência de relação entre a aplicação de fosfitos de potássio para o controle da murcha de verticilium (Verticillium dahliae Kleb.) e os mecanismos de defesa são citados em mudas de cacaueiro (17), onde não foi detectada indução na atividade de PO pela pulverização de fosfito de potássio na dose de 1,25 mL. $\mathrm{L}^{-1}$. No entanto, no mesmo estudo (17) tal dose foi fungitóxica, reduzindo a viabilidade de esporos do patógeno e o progresso da doença, corcordando com os resultados observados no presente estudo.

$\mathrm{O}$ aumento da atividade da FAL pode ser considerado como um indicador de resistência, pois esta enzima é requerida para a síntese de fenóis associados com a resistência (14). No entanto, para que essa hipótese seja válida, é importante que ocorra interação específica entre o patógeno e o hospedeiro, com a produção de compostos fenólicos e a sincronização da diversidade de respostas do hospedeiro no processo de infecção, o qual se relaciona com o tempo de desenvolvimento do patógeno (8). Tais enzimas têm a atividade determinada pela idade do tecido do hospedeiro assim como pela ação de indutores bióticos e abióticos $(8,16)$. No presente estudo, no entanto, tal comportamento não foi influenciado pelo uso dos fosfitos de potássio.

Estudos conduzidos na Itália mostraram a contaminação das maçãs por quantidades altas de ácido fosfórico nos pomares pulverizados com compostos dessa natureza (13). Resíduos de fosfitos foram detectados em maçãs quando as aplicações foram realizadas após a floração,

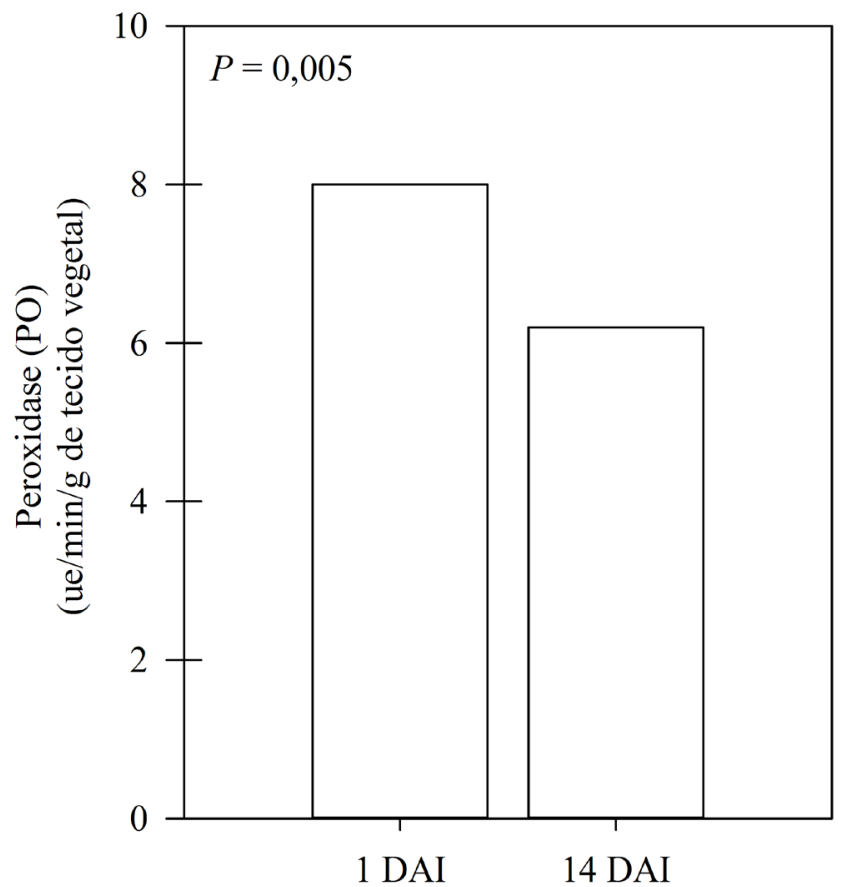

Dias após a inoculação (DAI)

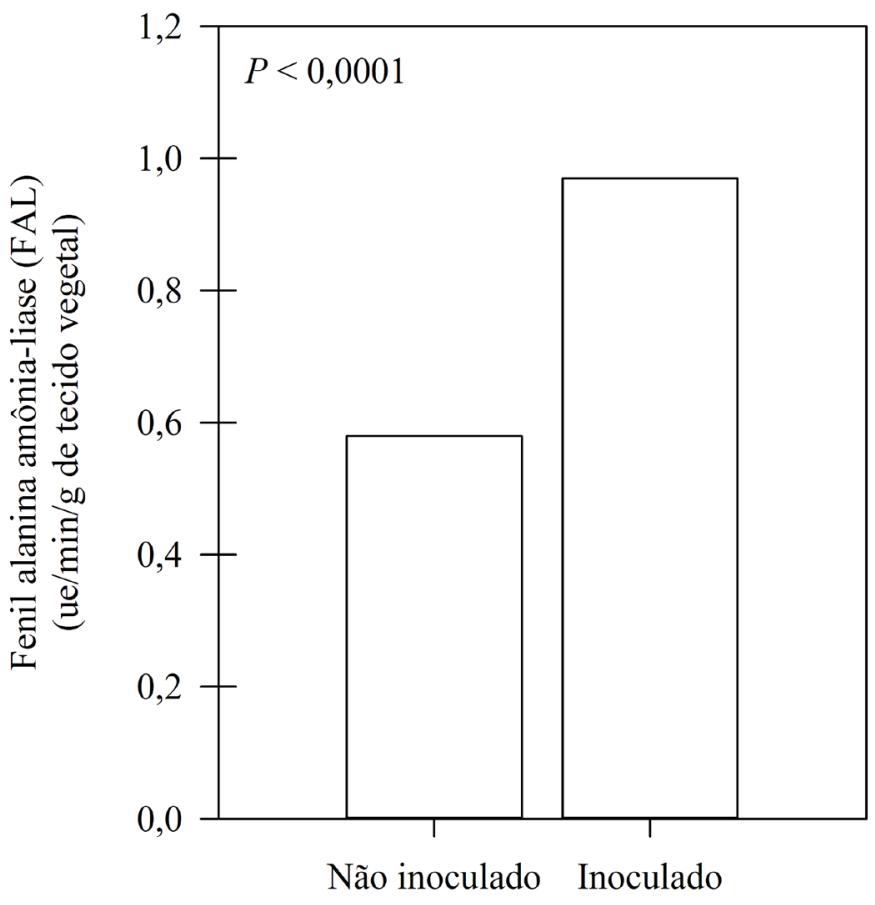

Inoculação com Cryptosporiopsis sp.

Figura 2. Atividade das enzimas peroxidase (PO) (à esquerda) em duas datas de amostragem, 1 dia após a inoculação (DAI) e 14 DAI (A) e fenilalanina amônialiase (à direita) em maçãs 'Pink Lady ${ }^{\circledR}$ ' não inoculadas ou inoculadas com Cryptosporiopsis sp. Vacaria, RS. 2007/08. 

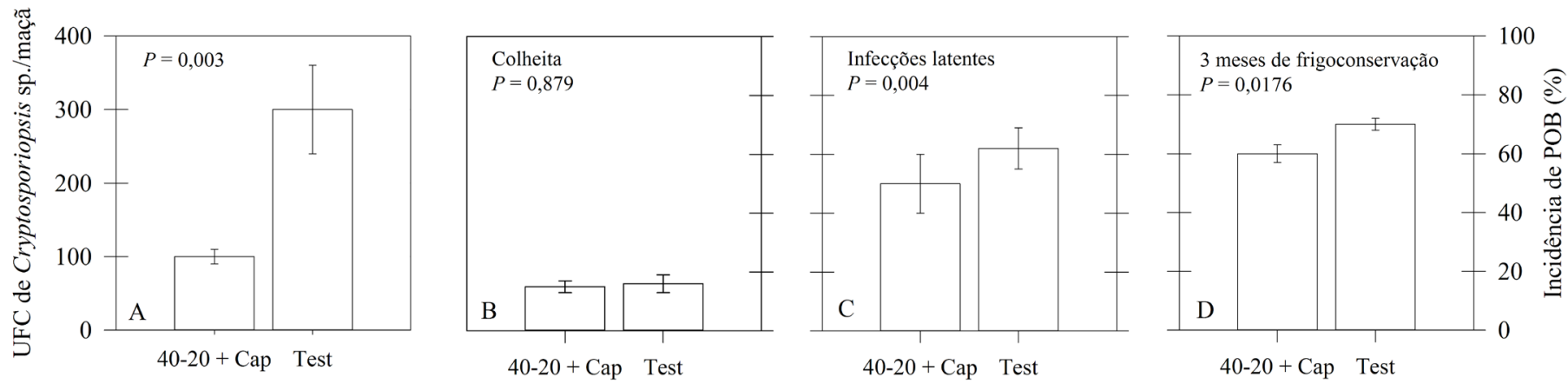

Tratamentos

Figura 3. Número de unidades formadoras de colônias (UFC) de Cryptosporiopsis sp. na superfície de maçãs 'Pink Lady ${ }^{\mathbb{B}}$ ' no momento da colheita (A) e incidência (\%) da podridão olho de boi no momento da colheita (B), infecções latentes (C) e após três meses de frigoconservação (D) em maçãs provenientes de parcelas pulverizadas com fosfito de potássio $\left(40 \% \mathrm{P}_{2} \mathrm{O}_{5}+20 \% \mathrm{~K}_{2} \mathrm{O}\right)$ em mistura com Captan (40-20 + Cap) e tratamento controle (Test). Pulverização realizada 24 h antes da colheita. Médias discriminadas pelo teste-t $(P<0,05)$. Vacaria, RS. 2007/08.

principalmente em aplicações na fase final de maturação dos frutos o que deve ser abordado em estudos futuros, principalmente no manejo da podridão olho de boi (13).

Pelos resultados do presente estudo, os fosfitos de potássio $(40 \%$ $\mathrm{P}_{2} \mathrm{O}_{5}$ e $\left.20 \% \mathrm{~K}_{2} \mathrm{O}\right)$ e $\left(30 \% \mathrm{P}_{2} \mathrm{O}_{5}\right.$ e $\left.20 \% \mathrm{~K}_{2} \mathrm{O}\right)$ avaliados apresentaram ação fungitóxica direta sobre os propágulos de $C p$., e o seu uso poderá ser recomendado em programas de manejo integrado da podridão olho de boi, principalmente pela capacidade de reduzir a população de propágulos do patógeno presente na superfície das maçãs. Em précolheita pode ser recomendado o seu uso em combinação com captan e, imediatamente anterior à colheita, como tática complementar no manejo da doença. Contudo, é recomendável a realização de estudos para avaliar o impacto da frequência de aplicação de sais de fosfito e o residual nos frutos nas condições do Brasil.

\section{AGRADECIMENTOS}

À Agropecuária Schio pela disponibilidade de área para a execução do experimento, aos funcionários do laboratório de fisiologia da Embrapa Clima Temperado pelo auxílio nas análises de atividade enzimática dos frutos, a CAPES e CNPq pela concessão de bolsas ao primeiro e segundo autores, respectivamente e ao Projeto Inova Maçã pelo suporte financeiro.

\section{REFERÊNCIAS}

1. Araújo, L.; Stadnik, M. J.; Borsato, L. C.; Valdebenito-Sanhueza, R. M. Fosfito de potássio e ulvana no controle da mancha foliar da Gala em macieira. Tropical Plant Pathology, Viçosa, v. 33, n. 2, p. 148-152, 2008.

2. Araújo, L.; Valdebenito-Sanhueza, R. M.; Stadnik, M. J. Avaliação de formulações de fosfto de potássio sobre Colletotrichum gloeosporioides in vitro e no controle pós-infeccional da mancha foliar de Glomerella em macieira. Tropical Plant Pathology, Viçosa, v. 35, n. 1, p. 54-59, 2010.

3. Belding, R. D.; Sutton, T. B.; Blakenship, S. M.; Young, E. Relationship between apple fruit epicuticular wax and growth of Peltaster fructicola and Leptodontidium elatius, two fungi that cause sooty blotch disease. Plant Disease, St. Paul, v. 84, n. 7, p. 767-772, 2000.

4. Blum, L. E. B.; Amarante, C. V. T.; Dezanet, A.; Lima, E. B.; Neto, P. H.; Ávila, R. D.; Siega, V. Fosfitos de potássio aplicados em pós-colheita reduzem o mofo-azul em maçãs 'Fuji' e 'Gala'. Revista Brasileira de Fruticultura, Jaboticabal, v. 29, n. 2, p. 265-268, 2007.

5. Brackmann, A.; Giehl, R. F. H.; Sestari, I.; Steffens, C. A. Fosfitos de potássio para o controle de podridões pós-colheita em maçãs 'Fuji' durante a fase de armazenamento refrigerado. Ciência Rural, Santa Maria, v. 34, n. 4, p. 1039-1042, 2004.

6. Costa, C.R. Determinação dos parâmetros (sólidos solúveis, pH e acidez titulaveis) em ameixas intactas usando espectromicroscopia no infravermelho próximo e seleção do comprimento de onda. Disponível em:http:// www.chemometricsufrn.org/uploads/1/2/7/2/12727894/dissertao_final_rosangela_cmara_ppgq.pdf.Acesso em 14/12/2014.

7. Campos, A. D.; Silveira, E. M. L. Metodologia para determinação da peroxidase e da polifenol oxidase em plantas. Pelotas: Embrapa Clima Temperado, 2003. (Comunicado Técnico, 87)

8. Campos, A. D.; Ferreira, A. G.; Hampe, M. M. V.; Antunes, I. F.; Brancão, N.; Silveira, E. P.; Osório, V. A.; Augustin, E. Atividade de peroxidase e polifenoxidase na resistência do feijão à antracnose. Pesquisa Agropecuária Brasileira, Brasília, DF, v. 39, n. 7, p. 637-643, 2004.

9. Gomes, G.L.G.C. Alterações metabólicas de plantas de milho submetidas à aplicação de glyphosate e fosfitos. Disponível em: http://www.pg.fca.unesp. br/Teses/PDFs/Arq0610.pdf. Acesso em: 14/12/2014.

10. Hyodo, H.; Yang, S.F. Ethylene enhanced synthesis of phenilalanineammonia-lyase in pea seedlings. Plant Physiology, Rockville, v. 47, n. 6, p. 765-770, 1971.

11. Hyodo, H.; Kuroda, H.; Yang, S.H. Introduction of PAL and increase in phenolics in lettuce in relation the development. Plant Physiology, Rockville, v. 62, p. 31-35, 1978.

12. World Health Organization. New York. IPCS - The international Programe on Chemical Safety. Disponível em: http://www.who.int/ipcs/ publications/training_poisons/basic_analytical_tox/en/index $8 . h t m l$. Acesso em: 03/12/2010

13. Kelderer, M.; Matteazzi, A.; Casera, C. Degradation behaviour of potassium K-phosphite in apple trees. In.: International Conference on Organic Fruit Growing, 13, 2008, Weinsberg. Anais. Weinsberg: Organic EPrint, 2008, p. 59-61.

14. Nicholson, R. L.; Hammerschmidt, H. Phenolic compounds and their role in disease resistance. Annual Review of Phytopathology, St. Paul, v. 30, p. 369-389, 1992.

15. Reuveni, M.; Harpaz, M.; Reuveni, R. Integrated control of powdery mildew on field-grow mango tree by foliar sprays of mono-potassium phosphate fertilizer, sterol inhibitor fungicides and the strobilurin kresoxym-methyl. European Journal of Plant Pathology, Dordrecht, v. 104, n. 9, p. 853-860, 1998.

16. Reuveni, M. Relationship between leaf age, peroxidase and $\beta-1,3-$ glucanase activity, and resistance to downy mildew in grapevines. Journal of Phytopathology, Berlim, v. 146, n. 10, p. 525-530, 1998.

17. Ribeiro Júnior, P. M.; Resende, M. L. V.; Pereira, R. B.; Cavalcanti, F. R.; Amaral, D. R.; Pádua, M. A. Fosfito de potássio na indução de resistência a Verticillium dahliae Kleb. em mudas de cacaueiro (Theobroma cacao L.). Ciência Agrotécnica, Lavras, v. 30, n. 4, p. 629-636, 2006.

18. Rosenberger, D. A.; Cox, K. D. Using phosphite fungicides to control apple diseases. New York Fruit Quarterly, Geneva, v. 17, p. 9-13, 2009.

19. Spolti, P.; Valdebenito-Sanhueza, R. M.; Del Ponte, E. M. Meio semiseletivo para recuperação e quantificação de Cryptosporiopsis perennans em maçãs. 
Ciência Rural, Santa Maria, v. 40, n. 3, p. 661-665, 2010.

20. Sutton, T. B.; Brown, E.; Meister, C. W. Efficacy of phosphorus-containing fungicides on summer diseases of apple, 2005. Fungicides and Nematicides Tests, St. Paul, v. 61, p. 4, 2005.

21. Valdebenito-Sanhueza R. M. Controle químico da podridão das raízes de macieira causada por Phytophthora cactorum no Rio Grande do Sul. Fitopatologia Brasileira, Brasília, v. 16, n. 1, p. 25-29, 1991.

22. Valdebenito-Sanhueza, R. M.; Maffioletti, M.; Comparin, C. C.; Krasniak, A.; Bogo, A.; Arcari, R. Características e controle da podridão 'olhode-boi' nas maçãs do Sul do Brasil. Bento Gonçalves: Embrapa Uva e Vinho. 2006. (Circular Técnica 66).
23. Valdebenito-Sanhueza, R. M.; Spolti, P.; Del Ponte, E. M. Controle do inóculo inicial para redução dos danos pela podridão: 'olho-de-boi' em macieiras. Revista Brasileira de Fruticultura, Jaboticabal, v. 32, n. 4, p. 1044-1054, 2010.

24. World Health Organization. WHOPES - WHO Pesticide Evaluation Scheme: "WHOPES". Disponível em: http://www.who.int/whopes/en/. Acesso em: 28/12/2010.

25. Wilkinson, C. J.; Holmes, J. M.; Dell, B.; Tynan, K. M.; McComb, B. L.; Shearer, I. J.; Colquhoun, G. E.; Hardy, S. T. J. Effect of phosphite on in planta zoospore production of Phytophthora cinnamoni. Plant Pathology, Oxford, v. 50, n. 5, p. 587-593, 2001. 